\title{
Pseudo-enzymatic hydrolysis of 4-nitrophenyl acetate by human serum albumin: pH-dependence of rates of individual steps
}

\author{
Paolo Ascenzi ${ }^{\mathrm{a}, \mathrm{b}, *}$, Magda Gioia ${ }^{\mathrm{c}, \mathrm{d}}$, Gabriella Fanali ${ }^{\mathrm{e}}$, Massimo Coletta ${ }^{\mathrm{c}, \mathrm{d}}$, Mauro Fasano ${ }^{\mathrm{e}}$ \\ a Interdepartmental Laboratory of Electron Microscopy, University Roma Tre, Via della Vasca Navale 79, I-00146 Roma, Italy \\ ${ }^{\mathrm{b}}$ National Institute of Biostructures and Biosystems, Viale Medaglie d'Oro 305, I-00136 Roma, Italy \\ ' Department of Clinical Sciences and Translational Medicine, University of Roma "Tor Vergata", Via Montpellier 1, I-00133 Roma, Italy \\ ${ }^{\mathrm{d}}$ Interuniversity Consortium for the Research on the Chemistry of Metals in Biological Systems, Via Celso Ulpiani 27, I-70126 Bari, Italy \\ ${ }^{\mathrm{e}}$ Department of Theoretical and Applied Sciences, Division of Biomedical Research, University of Insubria, Via Alberto da Giussano 12, I-21052 Busto Arsizio (VA), Italy
}

\section{A R T I C L E I N F O}

Article history:

Received 22 June 2012

Available online 4 July 2012

\section{Keywords:}

Human serum albumin

4-Nitrophenyl acetate

Pre-steady-state kinetics

Steady-state kinetics

$\mathrm{pH}$ effects

\begin{abstract}
A B S T R A C T
Human serum albumin (HSA) displays esterase activity reflecting multiple irreversible chemical modifications rather than turnover. Here, kinetics of the pseudo-enzymatic hydrolysis of 4-nitrophenyl acetate $(\mathrm{NphOAc})$ are reported. Under conditions where $[\mathrm{HSA}] \geqslant 5 \times[\mathrm{NphOAc}]$ and $[\mathrm{NphOAc}] \geqslant 5 \times[\mathrm{HSA}]$, the HSA-catalyzed hydrolysis of NphOAc is a first-order process for more than $95 \%$ of its course. From the dependence of the apparent rate constants $k_{\mathrm{app}}$ and $k_{\mathrm{obs}}$ on [HSA] and [NphOAc], respectively, values of $K_{\mathrm{s}}, k_{+2}$, and $k_{+2} / K_{\mathrm{s}}$ were determined. Values of $K_{\mathrm{s}}, k_{+2}$, and $k_{+2} / K_{\mathrm{s}}$ obtained at [HSA] $\geqslant 5 \times$ [NphOAc] and $[\mathrm{NphOAc}] \geqslant 5 \times[\mathrm{HSA}]$ are in good agreement, the deacylation step being rate limiting in catalysis. The $\mathrm{pH}$-dependence of $k_{+2} / K_{\mathrm{s}}, k_{+2}$, and $K_{\mathrm{s}}$ reflects the acidic $\mathrm{p} K_{\mathrm{a}}$ shift of the Tyr 411 catalytic residue from $9.0 \pm 0.1$ in the substrate-free HSA to $8.1 \pm 0.1$ in the HSA:NphOAc complex. Accordingly, diazepam inhibits competitively the HSA-catalyzed hydrolysis of NphOAc by binding to Tyr 411 .
\end{abstract}

(c) 2012 Elsevier Inc. All rights reserved.

\section{Introduction}

The three-domain organization of HSA is at the root of its capability to bind not only endogenous and exogenous low molecular weight compounds but also peptides and proteins at multiple sites. Remarkably, 35 proteins have been found to be associated to HSA under physiological conditions; the fraction of peptides and proteins bound to HSA has been defined as "albuminome" [1-6].

Upon binding endogenous and exogenous (macro)molecules, HSA undergoes chemical modifications, including acetylation, cysteinylation, homocysteinylation, glutathionylation, glycosylation, glycation, nitrosylation, nitration, oxidation, phosphorylation, biotinylation, and chlorination [6]; remarkably, chemical modifications may affect HSA binding properties and may confer antigenicity properties $[1,6,7]$.

In addition to its reversible and irreversible ligand binding capabilities, HSA displays pseudo-enzymatic properties. In fact, most of the apparent enzymatic properties of HSA (e.g., esterase

Abbreviations: AMPSO, 3-[(1,1-dimethyl-2-hydroxyethyl)amino]-2-hydroxypropanesulfonic acid; Bis-Tris, bis(2-hydroxyethyl)amino-tris(hydroxymethyl)methane; Hepes, 4-(2-hydroxyethyl)-1-piperazineethanesulfonic acid; HSA, human serum albumin; $\mathrm{NphOH}$, 4-nitrophenol; NphOAc, 4-nitrophenyl acetate.

* Corresponding author at: Interdepartmental Laboratory of Electron Microscopy, University Roma Tre, Via della Vasca Navale 79, I-00146 Roma, Italy. Fax: +39 06 57336321

E-mail address: ascenzi@uniroma3.it (P. Ascenzi). activity) are the result of multiple irreversible chemical modifications rather than of the catalytic activity occurring at a single reactive site [8]. Remarkably, the apparent HSA-catalyzed hydrolysis of 4-nitrophenyl acetate (NphOAc) is the result of the irreversible acetylation of 82 residues rather than of turnover. In fact, only Tyr411 is acetylated within the first $5 \mathrm{~min}$ of reaction with $5.0 \times 10^{-4} \mathrm{M}$ NphOAc; after $30 \mathrm{~min}$ to $6 \mathrm{~h}$, the partial acetylation of additional 16-17 residues of HSA takes place, including Asp1, Lys4, Lys12, Tyr411, Lys413, and Lys414. HSA incubation with $1.0 \times 10^{-2} \mathrm{M}$ NphOAc results in the acetylation of 59 Lys, $10 \mathrm{Ser}$, $8 \mathrm{Thr}, 4 \mathrm{Tyr}$, and 1 Asp [8].

Here, kinetics of the HSA pseudo-enzymatic hydrolysis of NphOAc, obtained under conditions where [HSA $] \geqslant 5 \times[\mathrm{NphOAc}]$ and $[\mathrm{NphOAc}] \geqslant 5 \times[\mathrm{HSA}]$ (between $\mathrm{pH} 5.8$ and 10.2 , at $22.0^{\circ} \mathrm{C}$ ), are reported. The $\mathrm{pH}$-dependence of the rates of the individual steps for the HSA pseudo-esterase activity probably depends on the acid-base equilibrium of Tyr411 and parallels the neutral-tobasic allosteric transition. Accordingly, diazepam inhibits competitively the HSA-catalyzed hydrolysis of NphOAc by binding to Tyr411.

\section{Materials and methods}

HSA, NphOAc, diazepam, 4-nitrophenol (NphOH), bis(2hydroxyethyl)amino-tris(hydroxymethyl)methane (Bis-Tris), 4-(2-hydroxyethyl)-1-piperazineethanesulfonic acid (Hepes), 3- 
[(1,1-dimethyl-2-hydroxyethyl)amino]-2-hydroxypropanesulfonic acid (AMPSO), and 2-amino-2-methyl-1-propanol were obtained from Sigma-Aldrich (St. Louis, MO, USA). All chemicals were of analytical or reagent grade and were used without further purification.

HSA (from Sigma-Aldrich, St. Louis, MO, USA) was essentially fatty acid free, according to the charcoal delipidation protocol [9-11], and was used without further purification. The HSA stock solution $\left([\mathrm{HSA}]=1.2 \times 10^{-2} \mathrm{M}\right.$ ) was prepared by dissolving HSA in $1.0 \times 10^{-2} \mathrm{M}$ phosphate buffer $\mathrm{pH} 7.0$, at $22.0^{\circ} \mathrm{C}$. The HSA concentration was determined spectrophotometrically at $279 \mathrm{~nm}$ $\left(\varepsilon=3.6 \times 10^{4} \mathrm{M}^{-1} \mathrm{~cm}^{-1}\right)$ [1]. Then, the HSA stock solution was diluted in the desired buffer (Bis-Tris buffer, $\mathrm{pH}$ 5.8-7.2; Hepes buffer, pH 6.8-8.2; AMPSO buffer, pH 8.3-9.7; 2-amino-2methyl-1-propanol buffer, $\mathrm{pH} 9.0-10.2$; all $0.1 \mathrm{M}$ ), the final $\mathrm{pH}$ ranging between 5.8 and 10.2. The final HSA concentration ranged between $4.0 \times 10^{-6} \mathrm{M}$ and $1.6 \times 10^{-3} \mathrm{M}$.

The NphOAc solution was prepared by dissolving the substrate in a $3.0 \times 10^{-3} \mathrm{M}$ Bis-Tris buffer solution ( $\mathrm{pH}$ 5.8) in the presence of $10 \%$ acetonitrile. The NphOAc concentration was determined spectrophotometrically at $400 \mathrm{~nm}\left(\varepsilon=1.8 \times 10^{4} \mathrm{M}^{-1} \mathrm{~cm}^{-1} ; \mathrm{pH}>\right.$ 8.5 and $22.0^{\circ} \mathrm{C}$ ), allowing to calculate the amount of 4-nitrophenol released from the substrate [12]. The final NphOAc concentration ranged between $1.0 \times 10^{-5} \mathrm{M}$ and $1.8 \times 10^{-3} \mathrm{M}$. The final acetonitrile concentration was $0.5 \%(\mathrm{v} / \mathrm{v})[13]$.

Kinetics and thermodynamics of the HSA-catalyzed hydrolysis of NphOAc were followed spectrophotometrically between $350 \mathrm{~nm}$ and $450 \mathrm{~nm}$ by mixing the HSA and NphOAc solutions with the SMF-20 rapid-mixing stopped-flow apparatus (Bio-Logic, Claix, France).

Kinetics and thermodynamics of the HSA-catalyzed hydrolysis of NphOAc, obtained under conditions where [NphOAc] $\geqslant 5 \times[\mathrm{H}-$ $\mathrm{SA}]$ and $[\mathrm{HSA}] \geqslant 5 \times[\mathrm{NphOAc}]$, between $\mathrm{pH} 5.8$ and 10.2 and $22.0^{\circ} \mathrm{C}$, were analyzed in the framework of the minimum three step-mechanism reported in Scheme $1[8,12-15]$, where HSA is the substrate-free protein, NphOAc is the substrate, HSA:NphOAc is the reversible protein-substrate complex, HSA-OAc is considered to be an ester formed between the acyl moiety of the substrate and the $\mathrm{O}$ atom of the Tyr411 phenoxyl group [8], AcOH is acetic acid, $k_{+1}$ is the second-order rate constant for the formation of the HSA:NphOAc complex starting from HSA and NphOAc, $k_{-1}$ is the first-order rate constant for the dissociation of the HSA:NphOAc complex to HSA and NphOAc, $K_{\mathrm{s}}\left(=k_{-1} / k_{+1}\right)$ is the pre-equilibrium constant, $k_{+2}$ is the first-order acylation rate constant, $k_{-2}$ is the first-order rate constant for the conversion of HSA-OAc to HSA:NphOAc, $k_{+3}$ is the first-order deacylation rate constant, and $k_{-3}$ is the second-order rate constant for the formation of the HSA-OAC adduct starting from $\mathrm{HSA}$ and $\mathrm{AcOH}$.

Kinetics and thermodynamics of the HSA-catalyzed hydrolysis of NphOAc, at $\mathrm{pH} 7.5$ and $22.0^{\circ} \mathrm{C}$, were also determined in the presence of diazepam ranging between $1.0 \times 10^{-5} \mathrm{M}$ and $7.7 \times 10^{-5} \mathrm{M}$. The HSA concentration was $4.0 \times 10^{-6} \mathrm{M}$ and the NphOAc concentration ranged between $1.0 \times 10^{-5} \mathrm{M}$ and $8.0 \times$ $10^{-5} \mathrm{M}[16]$.

Kinetics and thermodynamics of the HSA pseudo-esterase activity were analyzed using the GraphPad Prism program (GraphPad Software, Inc., La Jolla, CA, USA). The results are given as mean values of at least four experiments plus or minus the corresponding standard deviation.

$$
\begin{aligned}
& k_{+1} \quad k_{+2} \quad k_{+3} \\
& \mathrm{HSA}+\mathrm{NphOAc} \rightleftarrows \mathrm{HSA}: \mathrm{NpHOAc} \rightleftarrows \mathrm{HSA}-\mathrm{OAc} \rightleftarrows \mathrm{HSA}+\mathrm{AcOH} \\
& k_{-1} \quad k_{-2}+\mathrm{NphOH} \quad k_{-3}
\end{aligned}
$$

Scheme 1.

\section{Results and discussion}

The determination of kinetic parameters of Scheme 1 is simplified by the fact that the formation of the HSA:NphOAc complex from HSA and NphOAc may be regarded as being at equilibrium throughout the reaction (i.e., $k_{-1}>>k_{+2}$; see Scheme 1). This pseudo-first-order process is characterized by the rate constant $k_{1}{ }^{\text {obs }}$ given by Eq. (1):

$k_{1}^{o b s}=k_{+1} \times[\mathrm{HSA}]+k_{-1}$

Evidence for this is based on the observation that under all the present experimental conditions no lag phase occurs in the release of NphOH from NphOAc in the presence of HSA (see Fig. 1). This means that the equilibration of HSA:NphOAc with HSA and NphOAc is complete within $1.1 \mathrm{~ms}$ (i.e., the "dead-time" of the rapid-mixing stopped-flow apparatus), therefore $k_{1}{ }^{\text {obs }} \geqslant 3.5 \times$ $10^{3} \mathrm{~s}^{-1}$. Given that values of $K_{\mathrm{s}}\left(=k_{-1} / k_{+1}\right)$ for NphOAc binding to HSA range between $6.6 \times 10^{-5} \mathrm{M}$ and $5.1 \times 10^{-4} \mathrm{M}$ (see Table 1 ), it follows that $k_{-1} \geqslant 3.5 \times 10^{3} \mathrm{~s}^{-1}$ and $k_{+1} \geqslant 6.8 \times 10^{6} \mathrm{M}^{-1} \mathrm{~s}^{-1}$; therefore, since values of $k_{+2}$ range between $8.2 \times 10^{-3} \mathrm{~s}^{-1}$ and $2.1 \mathrm{~s}^{-1}$ (see Table 1 ), the prediction $k_{-1}>k_{+2}$ is satisfied. A further simplifying condition arises from the observation that the rate of $\mathrm{NphOH}$ release from NphOAc catalyzed by HSA is unaffected by the addition of $\mathrm{NphOH}$ (up to $1.0 \times 10^{-4} \mathrm{M}$ ) in the reaction mixtures, therefore values of $k_{-2}$ and $k_{-3}$ are very low, becoming indistinguinshable from $0 \mathrm{~s}^{-1}$. As a whole, the formation of the HSA:NphOAc complex from HSA and NphOAc can be treated as a rapid equilibrium process.

When $[\mathrm{HSA}] \geqslant 5 \times[\mathrm{NphOAc}]$ and $k_{-1}>>k_{+2}$, the rate of $\mathrm{NphOH}$ release from NphOAc should be a first-order process with a pseudo-first-order rate constant (i.e., $k_{\text {app }}$ ), according to Eq. (2):

$k_{\mathrm{app}}=\left(k_{+2} \times[\mathrm{HSA}]\right) /\left(K_{\mathrm{s}}+[\mathrm{HSA}]\right)$

The reaction of HSA with NphOAc is a first-order process for more than $95 \%$ of its course (Fig. 1, panel A) and values of $k_{\text {app }}$ are independent of the observation wavelength over the whole range explored (i.e., between 350 and $450 \mathrm{~nm}$ ) at fixed NphOAc concentration. Moreover, values of $k_{\text {app }}$ are independent of the NphOAc concentration when [HSA] $\geqslant 5 \times$ [NphOAc]. Values of $k_{+2}$ and $K_{\mathrm{s}}$ (see Table 1 ) were determined by Eq. (2) from hyperbolic plots of $k_{\text {app }}$ versus [HSA], as shown in Fig. 1 (panel B).

When $[\mathrm{NphOAc}] \geqslant 5 \times[\mathrm{HSA}]$, a mono-exponential time course preceding the apparent very slow pseudo-steady-state process occurs (Fig. 1, panel C). When $k_{+2} \geqslant 5 \times k_{+3}$, the differential equations arising from Scheme 1 may be solved [14] to describe the time course of $\mathrm{NphOH}$ release in the early stages of the reaction. The resulting expression is given in Eqs. (3)-(5):

$$
\begin{aligned}
{[\mathrm{NphOH}]=\left\{\left(k_{\text {cat }} \times[\mathrm{HSA}] \times[\mathrm{NphOAc}] \times t\right) /\left(K_{m}+[\mathrm{NphOAc}]\right)\right\} } \\
\\
+\alpha \times[\mathrm{HSA}] \times\left(1-e^{-k t}\right)
\end{aligned}
$$

where

$\alpha=\left\{\left(k_{+2} \times[\mathrm{NphOAc}]\right) /\left(\left(k_{+2}+k_{+3}\right) \times\left(K_{m}+[\mathrm{NphOAc}]\right)\right)\right\}^{2}$

and

$k_{\mathrm{obs}}=\left(k_{+2} \times[\mathrm{NphOAc}]\right) /\left(K_{s}+[\mathrm{NphOAc}]\right)+k_{+3}$

As predicted from Eqs. (3)-(5), a "burst" phase of $\mathrm{NphOH}$ release of amplitude $\alpha \times[\mathrm{HSA}]$ with the first-order rate constant $k_{\mathrm{obs}}$ occurs at all $\mathrm{pH}$ values explored. Values of $\alpha$, obtained at [NphOAc] $\geqslant 5 \times$ [HSA], range between 0.97 and 1.03 , the average $\alpha$ value is $1.00 \pm 0.03$. This indicates that the HSA:NphOAc: $\mathrm{NphOH}$ stoichiometry is $1: 1: 1$. Moreover, the time course of the "burst" phase of $\mathrm{NphOH}$ release is a first-order process for more than $95 \%$ of its course and values of $k_{\mathrm{obs}}$ are independent of the observation wavelength over the whole range explored (i.e., 

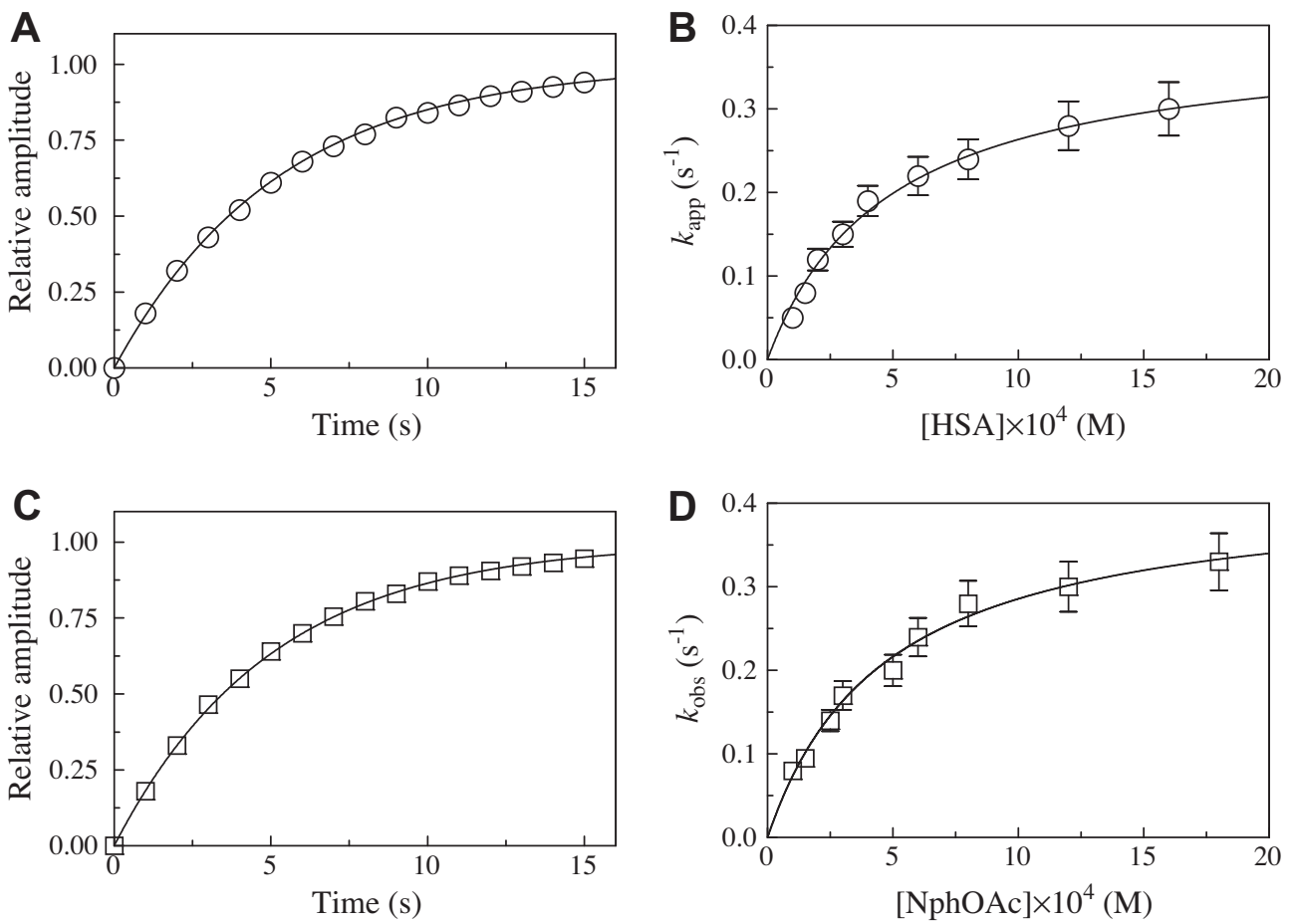

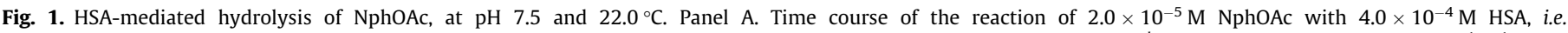

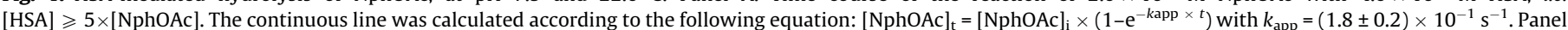

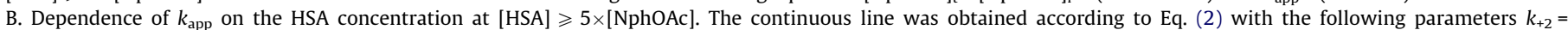

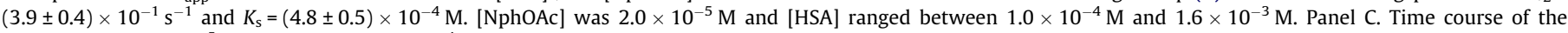

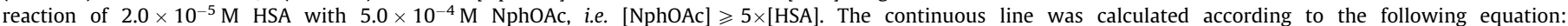

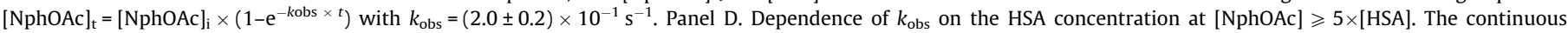

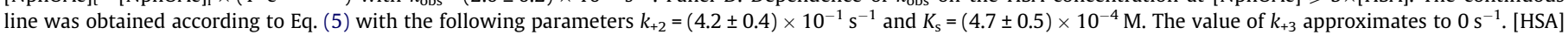
was $2.0 \times 10^{-5} \mathrm{M}$, and [NphOAc] ranged between $1.0 \times 10^{-4} \mathrm{M}$ and $1.8 \times 10^{-3} \mathrm{M}$. For details, see text.

Table 1

Values of catalytic parameters for the HSA-catalyzed hydrolysis of NphOAc, at $22.0^{\circ} \mathrm{C}$.

\begin{tabular}{|c|c|c|c|c|c|c|}
\hline \multirow[t]{2}{*}{$\mathrm{pH}$} & \multicolumn{3}{|c|}{$[\mathrm{HSA}] \geqslant 5 \times[\mathrm{NphOAc}]$} & \multicolumn{3}{|c|}{$[\mathrm{NphOAc}] \geqslant 5 \times[\mathrm{HSA}]$} \\
\hline & $K_{\mathrm{s}}(\mathrm{M})$ & $k_{+2}\left(\mathrm{~s}^{-1}\right)$ & $k_{+2} / K_{\mathrm{s}}\left(\mathrm{M}^{-1} \mathrm{~s}^{-1}\right)$ & $K_{\mathrm{s}}(\mathrm{M})$ & $k_{+2}\left(\mathrm{~s}^{-1}\right)$ & $k_{+2} / K_{\mathrm{s}}\left(\mathrm{M}^{-1} \mathrm{~s}^{-1}\right)$ \\
\hline 5.8 & $(4.3 \pm 0.4) \times 10^{-4}$ & $(8.2 \pm 0.8) \times 10^{-3}$ & $(1.9 \pm 0.2) \times 10^{1}$ & $(5.1 \pm 0.5) \times 10^{-4}$ & $(8.4 \pm 0.8) \times 10^{-3}$ & $(1.7 \pm 0.2) \times 10^{1}$ \\
\hline 6.8 & $(5.0 \pm 0.5) \times 10^{-4}$ & $(1.1 \pm 0.1) \times 10^{-1}$ & $(2.2 \pm 0.2) \times 10^{2}$ & $(4.8 \pm 0.5) \times 10^{-4}$ & $(1.0 \pm 0.1) \times 10^{-1}$ & $(2.0 \pm 0.2) \times 10^{2}$ \\
\hline 7.5 & $(4.8 \pm 0.5) \times 10^{-4}$ & $(3.9 \pm 0.4) \times 10^{-1}$ & $(8.1 \pm 0.9) \times 10^{2}$ & $(4.7 \pm 0.5) \times 10^{-4}$ & $(4.2 \pm 0.4) \times 10^{-1}$ & $(8.4 \pm 0.9) \times 10^{2}$ \\
\hline 8.0 & $(2.9 \pm 0.3) \times 10^{-4}$ & $(8.9 \pm 0.9) \times 10^{-1}$ & $(3.1 \pm 0.3) \times 10^{3}$ & $(3.1 \pm 0.3) \times 10^{-4}$ & $(8.5 \pm 0.8) \times 10^{-1}$ & $(3.0 \pm 0.3) \times 10^{3}$ \\
\hline 8.6 & $(1.5 \pm 0.2) \times 10^{-4}$ & $1.5 \pm 0.1$ & $(9.9 \pm 1.0) \times 10^{3}$ & $(1.3 \pm 0.1) \times 10^{-4}$ & $1.4 \pm 0.1$ & $(9.6 \pm 0.9) \times 10^{3}$ \\
\hline 9.0 & $(1.1 \pm 0.1) \times 10^{-4}$ & $1.9 \pm 0.2$ & $(1.7 \pm 0.2) \times 10^{4}$ & $(1.0 \pm 0.1) \times 10^{-4}$ & $2.1 \pm 0.2$ & $(1.9 \pm 0.2) \times 10^{4}$ \\
\hline 9.6 & $(7.7 \pm 0.8) \times 10^{-5}$ & $2.1 \pm 0.2$ & $(2.7 \pm 0.3) \times 10^{4}$ & $(7.5 \pm 0.7) \times 10^{-5}$ & $1.9 \pm 0.2$ & $(2.8 \pm 0.3) \times 10^{4}$ \\
\hline 10.2 & $(6.6 \pm 0.7) \times 10^{-5}$ & $2.0 \pm 0.2$ & $(3.2 \pm 0.3) \times 10^{4}$ & $(6.8 \pm 0.7) \times 10^{-5}$ & $2.1 \pm 0.2$ & $(3.1 \pm 0.3) \times 10^{4}$ \\
\hline
\end{tabular}

between 350 and $450 \mathrm{~nm}$ ) at fixed HSA concentration. Values of $k_{\text {obs }}$ are independent of the HSA concentration when [NphOAc] $\geqslant 5 \times[\mathrm{HSA}]$. Values of $k_{+2}$ and $K_{\mathrm{s}}$ (see Table 1 ) were determined by Eq. (5) from hyperbolic plots of $k_{\mathrm{obs}}$ versus [S], as shown in Fig. 1 (panel D). Under all the experimental conditions, the $y$-intercept of the hyperbola described by Eq. (5) approximates to $0 \mathrm{~s}^{-1}$, thus indicating that the value of $k_{+3}$ is at least 100-fold smaller than the $k_{\text {obs }}$ value obtained at the lowest NphOAc concentration (i.e., $k_{+3}<10^{-5} \mathrm{~s}^{-1}$ ). Accordingly, $k_{+3}$ for Tyr411$\mathrm{OAc}$ deacylation corresponds to $3.2 \times 10^{-6} \mathrm{~s}^{-1}$, at $\mathrm{pH} 8.0$ and $22.0^{\circ} \mathrm{C}[8]$.

As predicted by Scheme 1, values of $K_{\mathrm{s}}$ and $k_{+2}$ obtained under conditions where $[\mathrm{HSA}] \geqslant 5 \times[\mathrm{NphOAc}]$ from Eq. (2) are in excellent agreement with those obtained under conditions where $[\mathrm{NphOAc}] \geqslant 5 \times[\mathrm{HSA}]$ from Eq. (5) (see Table 1 ). Moreover, data here reported indicate that the deacylation process is rate limiting in the HSA-catalyzed hydrolysis of NphOAc (i.e., $k_{+3}<<k_{+2}$ ). Lastly, values of $K_{\mathrm{s}}$ and $k_{+2}$ here obtained are in agreement with those previously reported $[12,13]$

Accounting for values of $K_{\mathrm{s}}=2.9 \times 10^{-4} \mathrm{M}$ (present study), $k_{+2}=8.9 \times 10^{-1} \mathrm{~s}^{-1}$ (present study), and $k_{+3}=3.2 \times 10^{-6} \mathrm{~s}^{-1}$ [8], values of $K_{\mathrm{m}}\left(\cong 1 \times 10^{-9} \mathrm{M}\right)$ and $k_{\text {cat }}\left(\cong 3 \times 10^{-6} \mathrm{~s}^{-1}\right)$ for the HSAcatalyzed hydrolysis of NphOAc have been estimated at $\mathrm{pH} 8.0$ and $22.0^{\circ} \mathrm{C}$, according to Eqs. (6) and (7):

$K_{m}=\left(K_{s} \times k_{+3}\right) /\left(k_{+2}+k_{+3}\right)$

$k_{\text {cat }}=\left(k_{+2} \times k_{+3}\right) /\left(k_{+2}+k_{+3}\right)$

Notably, values of $K_{\mathrm{m}}$ and $k_{\text {cat }}$ for the HSA-catalyzed hydrolysis of NphOAc cannot be determined experimentally since during the very low turnover (i.e., $k_{\text {cat }} \cong 3 \times 10^{-6} \mathrm{~s}^{-1}$ ) 81 amino-acid residues undergo acetylation, under conditions where [NphOAc] >> [HSA] [8]. 

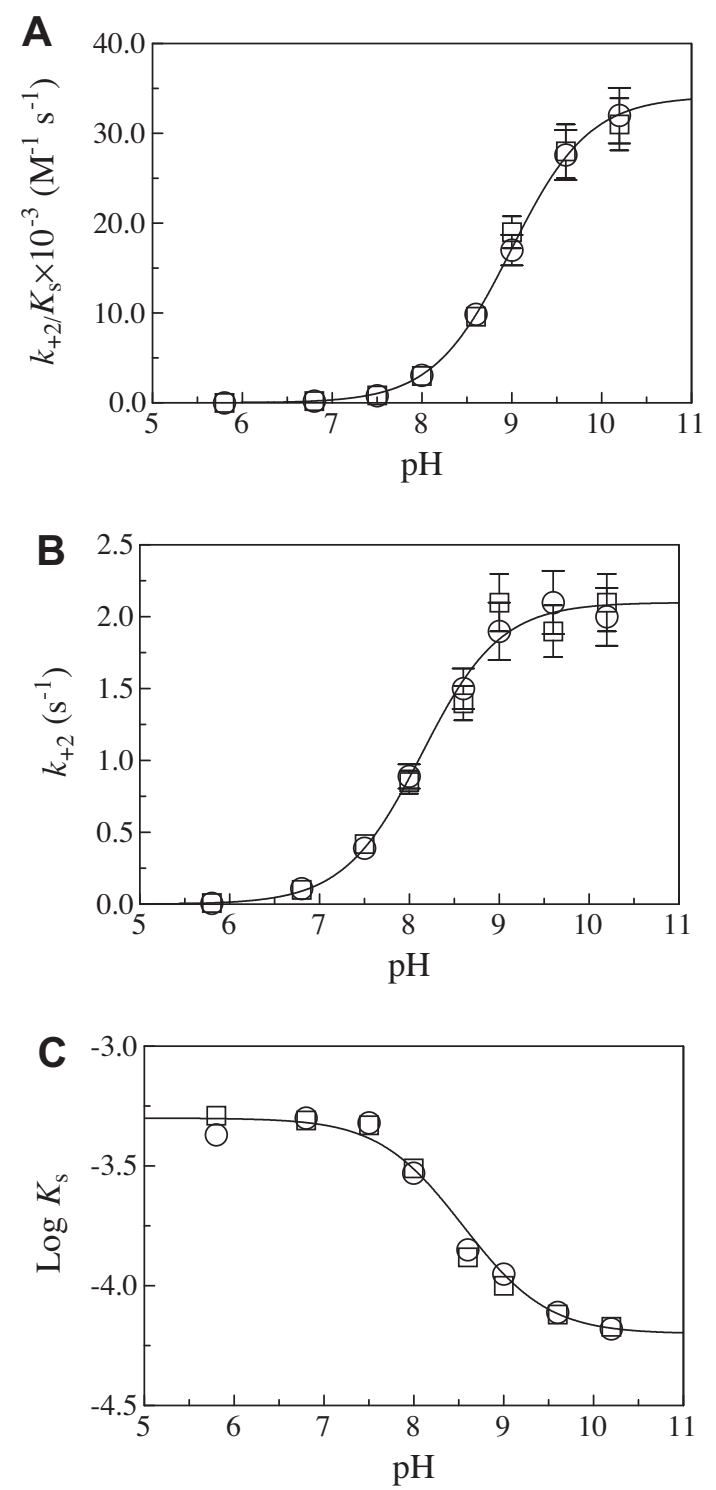

Fig. 2. pH-dependence of $k_{+2} / K_{\mathrm{s}}$ (panel A), $k_{+2}$ (panel B), and $K_{\mathrm{s}}$ (panel C) for HSATyr411-mediated hydrolysis of NphOAc, at $22.0^{\circ} \mathrm{C}$. Circles and squares indicate data obtained under conditions where $[\mathrm{HSA}] \geqslant 5 \times[\mathrm{NphOAc}]$ and $[\mathrm{NphOAc}] \geqslant$ $5 \times[\mathrm{HSA}]$, respectively. The continuous lines were obtained according to Eqs. (8)(10) with the following parameters: panel A - p $K_{\mathrm{unl}}=9.0 \pm 0.1$ and $\left(k_{+2} / K_{\mathrm{s}}\right)^{\mathrm{lim}}=$ $(3.4 \pm 0.4) \times 10^{4} \mathrm{M}^{-1} \mathrm{~s}^{-1}$; panel B $-\mathrm{p} K_{\text {lig }}=8.1 \pm 0.1$ and $k_{+2}^{\lim }=2.1 \pm 0.2 \mathrm{~s}^{-1}$; and panel $\mathrm{C}-\mathrm{p} K_{\mathrm{unl}}=9.0 \pm 0.1, \mathrm{p} K_{\mathrm{lig}}=8.1 \pm 0.1$, and $K_{\mathrm{s}}^{\lim }=(6.6 \pm 0.7) \times 10^{-5} \mathrm{M}$. For details, see text.

Fig. 2 shows the $\mathrm{pH}$-dependence of $k_{+2} / K_{\mathrm{s}}, k_{+2}$, and $K_{\mathrm{s}}$ values for the HSA-catalyzed hydrolysis of NphOAc. Values of kinetic parameters obtained using different buffers at overlapping $\mathrm{pH}$ values match each other within the experimental error. Values of $\mathrm{p} K_{\mathrm{a}}$ modulating the $\mathrm{pH}$-dependence of $k_{+2} / K_{\mathrm{s}}, k_{+2}$, and $K_{\mathrm{s}}$ were determined by data analysis according to Eqs. (8)-(10):

$$
\begin{aligned}
& \log K_{s}=-\log K_{s}^{l i m}+\log \left(\left(10^{-p H}+10^{-p K u n l}\right) /\left(10^{-p H}+10^{-p K l i g}\right)\right) \\
& k_{+2}=k_{+2}^{\lim } /\left(1+\left(10^{-p H} / 10^{-p K l i g}\right)\right) \\
& k_{+2} / K_{s}=\left(k_{+2} / K_{s}\right)^{\lim } /\left(1+\left(10^{-p H} / 10^{-p K u n l}\right)\right)
\end{aligned}
$$

where $K_{s}^{\lim }, k_{2}^{\lim }$, and $\left(k_{+2} / K_{s}\right)^{\text {lim }}$ are the alkaline asymptotes of $K_{\mathrm{s}}, k_{+2}$, and $k_{+2} / K_{s}$.
According to linked functions [14-16], the pH-dependence of $k_{+2} / K_{\mathrm{s}}$ and $k_{+2}$ reflects the acid-base equilibrium of a single amino-acid residue in the substrate-free HSA (i.e., $\mathrm{p} K_{\mathrm{unl}}=9.0 \pm 0.1$ ) and in the HSA:NphOAc complex (i.e., $\mathrm{p} K_{\text {lig }}=8.1 \pm 0.1$ ), respectively. Moreover, the $\mathrm{pH}$-dependence of $K_{\mathrm{s}}$ reflects the acidic $\mathrm{p} K_{\mathrm{a}}$ shift of a single amino-acid residue from the substrate-free HSA (i.e., $\mathrm{p} K_{\mathrm{unl}}=9.0 \pm 0.1$ ) to the HSA:NphOAc complex (i.e., $\mathrm{p} K_{\mathrm{lig}}=$ $8.1 \pm 0.1)$. The $\mathrm{p} K_{\mathrm{unl}}$ value here reported is in agreement with that given in the literature $[12,13]$. As expected, the $\mathrm{p} K_{\text {unl }}$ value is substrate-independent (see present study and [17]); on the other hand, the $\mathrm{p} K_{\text {lig }}$ value depends on the chemical structure of the substrate (see present study and [17]), reflecting the different solvent accessibility of the ionization group(s) modulating catalysis.

The acidic $\mathrm{p} K_{\mathrm{a}}$ shift of an apparently single ionizable side chain group of HSA upon NphOAc binding could reflect the reduced solvent accessibility of Tyr411, representing the primary esterase site of HSA (see $[8,13,17])$, although long range effects could not be excluded.

Tyr 411 is located in the FA3-FA4 cleft that is made of a mainly apolar region forming the FA3 site and a polar patch contributing the FA4 site. The polar patch is centered on Tyr411 and includes Arg410, Lys414, and Ser489 [5,15]. The analysis of the threedimensional structure of the ligand-free HSA [19] and of the molecular model of the HSA:4-nitrophenyl propionate complex [13] suggests that the observed pH effects (Fig. 2) could reflect the acidic $\mathrm{p} K_{\mathrm{a}}$ shift of Tyr411. This would render more stable the negative charge on the phenoxyl oxygen atom of Tyr411, which indeed appears to form a hydrogen bond with the carbonyl oxygen atom of 4-nitrophenyl propionate [13], potentiating its nucleophilic role as an electron donor in the pseudo-esterase activity of HSA.

In order to highlight the role of Tyr411, located in the FA3-FA4 cleft [18], as the primary esterase site for the HSA-catalyzed hydrolysis of NphOAc $[8,13,17]$, the inhibitory effect of diazepam has been investigated under conditions where [NphOAc] $>[\mathrm{HSA}]$. In fact, diazepam binds at the center of the FA3-FA4 cleft with one oxygen atom interacting with the hydroxyl group of Tyr411 [18]. As expected for the pure competitive inhibition mechanism [20], values of $K_{\mathrm{s}}$ for the HSA-catalyzed hydrolysis of NphOAc increase with the diazepam concentration (i.e., [I]; Fig. 3), whereas values of $k_{+2}$ are unaffected by the drug. The analysis of the linear dependence of the $K_{s}^{a p p} / K_{s}$ ratio on the diazepam concentration (i.e., [I]) according to Eq. (11):

$K_{s}^{\text {app }} / K_{s}=[I] / K_{I}+1$

allowed to determine the value of the equilibrium constant for diazepam binding to HSA (i.e., $K_{\mathrm{I}}$, corresponding to the absolute value of

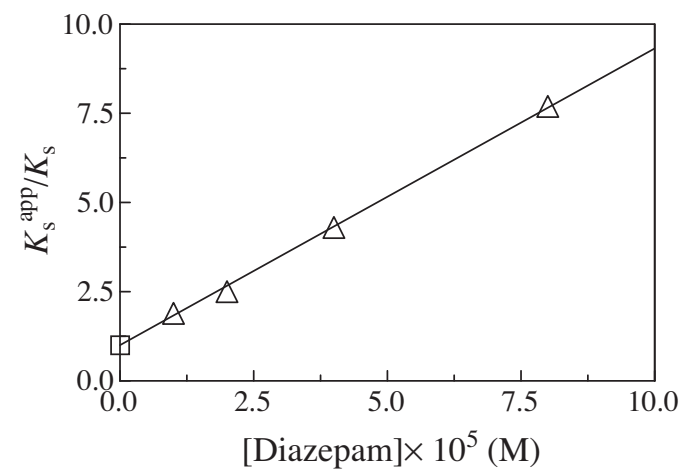

Fig. 3. Competitive inhibitory effect of diazepam on the HSA-catalyzed hydrolysis of NphOAc, at pH 7.5 and $22.0^{\circ} \mathrm{C}$. Data were obtained under conditions where $[\mathrm{NphOAc}]>[\mathrm{HSA}]$. The continuous line was obtained according to Eq. (11) with $K_{\mathrm{I}}=1.2 \times 10^{-5} \mathrm{M}$. For details, see text. 
the $x$ intercept of the linear plot). The value of $K_{\mathrm{I}}$ here determined agrees with that reported previously $[17,21]$. These data agree with the view that Tyr411 located at the FA3-FA4 cleft [18] is the primary esterase site for the HSA-catalyzed hydrolysis of NphOAc $[8,13]$.

\section{Conclusion}

The reaction of NphOAc with HSA is reminiscent of that observed for acylating agents with other proteins (e.g., cysteine and serine proteinases) [14,22]. However, the HSA-catalyzed hydrolysis of NphOAc is the result of the irreversible acetylation of 82 residues rather than of turnover [8]. Remarkably, the reaction of Tyr411 with NphOAc proceeds much faster than that catalyzed by any other amino-acid side chain of HSA $[8,13]$.

Present data support the following considerations: (i) The hydrolysis of NphOAc by Tyr411, i.e. the most reactive group of HSA, appears to involve strong reversible binding prior to reaction. (ii) NphOAc acts as a suicide substrate, the value of the deacylation rate constant (i.e., $k_{+3}$ ) being lower by several orders of magnitude than that of the acylation rate constant (i.e., $k_{+2}$ ). (iii) The hydrolysis of NphOAc by HSA is inhibited competitively by diazepam, since both the substrate and the drug interact with Tyr411 at the FA3FA4 cleft. (iv) The ionization state of Tyr411 modulates NphOAc hydrolysis; indeed substrate binding to HSA induces the acidic $\mathrm{p} K_{\mathrm{a}}$ shift of the Tyr411 residue. (v) HSA acylation appears relevant from the pharmacokinetic viewpoint; indeed HSA acylation by aspirin [23], beside increasing the affinity of phenylbutazone and inhibiting bilirubin binding, reduces prostaglandin affinity, accelerating the clearance of prostaglandins and serving as an additional mechanism of the aspirin anti-inflammatory effect [24].

\section{Acknowledgments}

This work was partially supported by a grant from Ministero dell'Istruzione, dell'Università e della Ricerca of Italy (Università Roma Tre, Roma, Italy; CLAR 2011 to P.A.).

\section{References}

[1] T. Peters Jr. (Ed.), All about Albumin: Biochemistry, Academic Press, San Diego and London, Genetics and Medical Applications, 1996.

[2] S. Curry, Beyond expansion: structural studies on the transport roles of human serum albumin, Vox Sang 83 (Suppl. 1) (2002) 315-319.

[3] M. Fasano, S. Curry, E. Terreno, M. Galliano, G. Fanali, P. Narciso, S. Notari, P. Ascenzi, The extraordinary ligand binding properties of human serum albumin, IUBMB Life 57 (2005) 787-796.
[4] R.L. Gundry, Q. Fu, C.A. Jelinek, J.E. Van Eyk, R.J. Cotter, Investigation of an albumin-enriched fraction of human serum and its albuminome, Proteomics Clin. Appl. 1 (2007) 73-88.

[5] S. Curry, Lessons from the crystallographic analysis of small molecule binding to human serum albumin, Drug Metab. Pharmacokinet. 24 (2009) 342-357.

[6] G. Fanali, A. di Masi, V. Trezza, M. Marino, M. Fasano, P. Ascenzi, Human serum albumin: from bench to bedside, Mol. Aspects Med. 33 (2012) 209-290.

[7] K. Honma, M. Nakamura, Y. Ishikawa, Acetylsalicylate-human serum albumin interaction as studied by NMR spectroscopy-antigenicity-producing mechanism of acetylsalicylic acid, Mol. Immunol. 28 (1991) 107-113.

[8] O. Lockridge, W. Xue, A. Gaydess, H. Grigoryan, S.J. Ding, L.M. Schopfer, S.H. Hinrichs, P. Masson, Pseudo-esterase activity of human albumin: slow turnover on tyrosine 411 and stable acetylation of 82 residues including 59 lysines, J. Biol. Chem. 283 (2008) 22582-22590.

[9] R.F. Chen, Removal of fatty acids from serum albumin by charcoal treatment, J. Biol. Chem. 242 (1967) 173-181.

[10] M. Sogami, J.F. Foster, Isomerization reactions of charcoal-defatted bovine plasma albumin. The N-F transition and acid expansion, Biochemistry 7 (1968) 2172-2182.

[11] J. Cabrera-Crespo, V.M. Goncalves, E.A. Martins, S. Grellet, A.P. Lopes, I. Raw, Albumin purification from human placenta, Biotechnol. Appl. Biochem. 31 (2000) 101-106.

[12] G.E. Means, M.L. Bender, Acetylation of human serum albumin by $p$ nitrophenyl acetate, Biochemistry 14 (1975) 4989-4994.

[13] Y. Sakurai, S.F. Ma, H. Watanabe, N. Yamaotsu, S. Hirono, Y. Kurono, U. KraghHansen, M. Otagiri, Esterase-like activity of serum albumin: characterization of its structural chemistry using $p$-nitrophenyl esters as substrates, Pharm. Res. 21 (2004) 285-292.

[14] M.R. Hollaway, E. Antonini, M. Brunori, The pH-dependence of rates of individual steps in ficin catalysis, Eur. J. Biochem. 24 (1971) 332-341.

[15] E. Antonini, P. Ascenzi, The mechanism of trypsin catalysis at low pH: proposal for a structural model, J. Biol. Chem. 256 (1981) 12449-12455.

[16] L. Peller, R.A. Alberty, Multiple intermediates in steady state enzyme kinetics: I. The mechanism involving a single substrate and product, J. Am. Chem. Soc. 81 (1959) 5907-5914.

[17] P. Ascenzi, M. Fasano, Pseudo-enzymatic hydrolysis of 4-nitrophenyl myristate by human serum albumin, Biochem. Biophys. Res. Commun. 422 (2012) 219223.

[18] J. Ghuman, P.A. Zunszain, I. Petitpas, A.A. Bhattacharya, M. Otagiri, S. Curry, Structural basis of the drug-binding specificity of human serum albumin, J. Mol. Biol. 353 (2005) 38-52.

[19] S. Sugio, A. Kashima, S. Mochizuki, M. Noda, K. Kobayashi, Crystal structure of human serum albumin at 2.5 Å resolution, Protein Eng. 12 (1999) 439-446.

[20] P. Ascenzi, M.G. Ascenzi, G. Amiconi, Enzyme competitive inhibition. Graphical determination of $K_{\mathrm{i}}$ and presentation of data in comparative studies, Biochem. Mol. Biol. Edu. 15 (1987) 134-135.

[21] G. Fanali, Y Cao, P. Ascenzi, V. Trezza, T. Rubino, D. Parolaro, M. Fasano, Binding of 89 -tetrahydrocannabinol and diazepam to human serum albumin, IUBMB Life 63 (2011) 446-451.

[22] F.J. Kezdy, M.L. Bender, The kinetics of the $\alpha$-chymotrypsin-catalyzed hydrolysis of p-nitrophenyl acetate, Biochemistry 1 (1962) 1097-1106.

[23] F. Yang, C. Bian, L. Zhu, G. Zhao, Z. Huang, M. Huang, Effect of human serum albumin on drug metabolism: structural evidence of esterase activity of human serum albumin, J. Struct. Biol. 157 (2007) 348-355.

[24] M.S. Liyasova, L.M. Schopfer, O. Lockridge, Reaction of human albumin with aspirin in vitro: mass spectrometric identification of acetylated lysines 199, 402, 519, and 545, Biochem. Pharmacol. 79 (2010) 784-791. 